\title{
Hypoxia suffocates histone demethylases to change gene expression: a metabolic control of histone methylation
}

\author{
Hyunsung Park ${ }^{*}$ \\ Department of Life Science, University of Seoul, Seoul 02504, Korea
}

\begin{abstract}
Hypoxia affects various physiological and pathophyological processes. Hypoxia changes the expression of hypoxiaresponsive genes through two main pathways. First, hypoxia activates transcription factors (TF) such as Hypoxia-inducible Factor (HIF). Second, hypoxia decreases the activity of Jumonji $\mathrm{C}$ domain-containing histone demethylases (JMJDs) that require $\mathrm{O}_{2}$ and $\alpha$-Ketoglutarate $(\alpha-\mathrm{KG})$ as substrates. The JMJDs affect gene expression through their regulation of active or repressive histone methylations. Profiling of H3K4me3, H3K9me3, and H3K27me3 under both normoxia and hypoxia identified 75 TFs whose binding motifs were significantly enriched in the methylated regions of the genes. TFs showing similar binding strengths to their target genes might be under the 'metabolic control' which changes histone methylation and gene expression by instant changing catalytic activities of resident histone demethylases. [BMB Reports: Perspective 2017; 50(11): 537-538]
\end{abstract}

The transcription Factor (TF) determines which subset of genes should be induced in response to intra and extracellular signals. Many studies have focused on the mechanisms of how TFs respond to specific signals, bind specific DNA sequences and recruit molecular machines on a right base pair where

*Corresponding author. E-mail: ksuk@knu.ac.kr

https://doi.org/10.5483/BMBRep.2017.50.11.200

Received 30 September 2017

Keywords: ChIP-seq, Histone demethylases, Hypoxia, Metabolic control, Transcription factors

Abbreviations: $\alpha-K G, \alpha$-Ketoglutarate; ChIP-seq, Chromatin immnuoprecipetation-sequencing; DEG, differentially expressed gene; DMR, differentially methylated genes; $\mathrm{H} 3 \mathrm{~K} 4 \mathrm{me} 3$, tri-methylation of $4^{\text {th }}$ lysine residue of histone 3; JMJD, Jumonji C domain-containing histone demethylase; TF, Transcription Factor; TSS, transcription start site

Perspective to: Seongyeol Lee (2017) Multi-dimensional histone methylations for coordinated regulation of gene expression under hypoxia. Nucleic Acids Research Accepted online 2017, August 25 https://doi.org/10.1093/nar/gkx747 transcription should start. Many signaling pathways alter gene expression by regulating the function of the TFs: this includes their abundance; covalent modification; nuclear translocation; protein-protein interaction or conformation. Epigenetic studies have revealed that covalent modifications of DNA and histones are strongly correlated with gene expression. Considering the types of histones $(\mathrm{H} 1, \mathrm{H} 2 \mathrm{~A}, \mathrm{H} 2 \mathrm{~B}, \mathrm{H} 3, \mathrm{H} 4$ etc.), the numbers of amino acid residues which can be covalently modified, the types of modifications (methylation, acetylation, phosphorylation, ubiquitination, etc.), and the relative locations of histones from a gene (promoter, enhancer, coding region, transcription start site (TSS), intergenic region), the combination of histone modifications (i.e. histone codes) is enormously diverse. Histone methylation affects gene expression in distinct localized patterns. The trimethylation of the $4^{\text {th }}$ lysine residue of histone $3(\mathrm{H} 3 \mathrm{~K} 4 \mathrm{me} 3)$ are found at TSS in active genes. $\mathrm{H} 3 \mathrm{~K} 9 \mathrm{me} 3$ and H3K27me3 are often found in repressed genes within a heterochromatin region. The histones are ornamented with diverse modifications by enzymes, so called 'writers'; they are also removed by the opposite enzymes, so called 'erasers'. These histone modifiers do not have DNA binding domains; thus, they are unable to target specific histones which were wrapped with distinct DNA sequences along the chromatins. The DNA-bound TFs (or DNA bound long noncoding RNA) recruit the histone modifiers through a protein-protein interaction. Therefore, the TFs assign specifically localized histones to the histone modifiers.

Most of the histone demethylases contain Jumonji C (Jmj C) domains in their catalytic region. The Jmj C-domain containing histone demethylases (JMJD/JHDM) catalyze the oxidative demethylation of a methylated lysine residue of histones by using $\mathrm{O}_{2}, \alpha$-ketoglutarate $(\alpha-K G)$, vitamin $\mathrm{C}$, and $\mathrm{Fe}(\mathrm{II})$. The histone demethylases have different substrate specificities. JMJD1A demethylates H3K9me2 and me1, JMJD2 isozymes demethylate H3K9me3, Jarid1 isozymes demethylate H3K4me3, and JMJD3 demethylates H3K27me3. The findings that JMJDs need $\mathrm{O}_{2}, \alpha-K G$ and reducing agents suggest that the metabolic changes in $\mathrm{O}_{2}, \alpha-K G$ and redox status instantly alter the activities of JMJDs. These instant metabolic changes bypass the signaling pathways which are required for activating the TFs, but instead, they just alter the catalytic activities of the JMJDs, which are already assigned to specific histones through their interactions with the DNA-binding TFs. This direct 'metabolic

ISSN: 1976-670X (electronic edition)

Copyright (c) 2017 by the The Korean Society for Biochemistry and Molecular Biology

(c) This is an open-access article distributed under the terms of the Creative Commons Attribution Non-Commercial License (http://creativecommons.org/licenses/by-nc/4.0) which permits unrestricted non-commercial use, distribution, and reproduction in any medium, provided the original work is properly cited. 

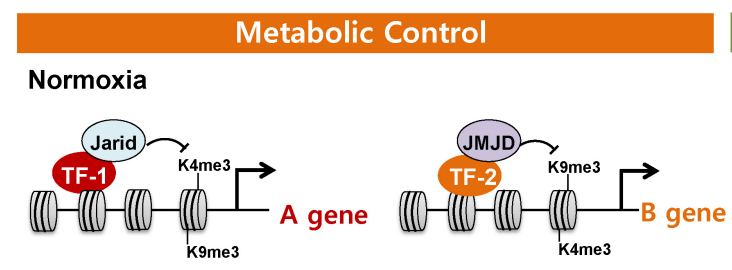

Hypoxia
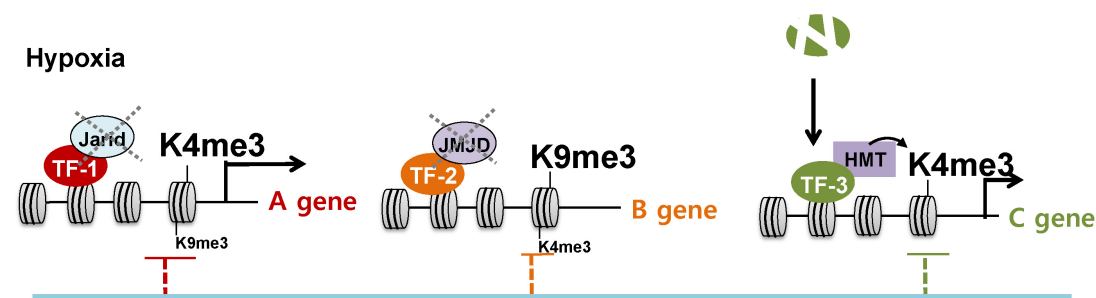

MotifLocator Analyses: Consensus Seq of DMR, prediction of possible TFs
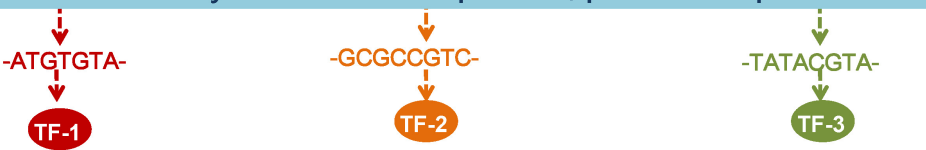

\begin{abstract}
Diagram 1. Schematic model for metabolic control and signaling control in response to hypoxia. Hypoxia increases only single type of histone methylation (either H3K4me3 or H3K9me3 are shown). By the MotifLocator analyses, we predicted 75 TFs which have their DNA binding sequences on DMRs. Two mechanisms, metabolic control and signaling control can explain how hypoxia regulates gene expression by changing the histone methylation.
\end{abstract}

control' of gene expression is in contrast with the conventional 'signaling control' of gene expression, which consists of multistep signal transduction processes for conveying extracellular signals to the specific transcription factors (TFs) (Diagram 1).

We performed a global profiling of H3K4me3, H3K9me3, and H3K27me3 in human adipocyte-derived stem cells under both normoxia and hypoxia. Further, we identified differentially methylated genes (DMGs) by hypoxia for each type of the histone methylation. We also performed gene expression profiling and identified differentially expressed genes (DEGs) by hypoxia. Surprisingly, the DEGs involved predominant changes in a single type of histone methylation, rather than a complex combinatorial change of multiple types of histone methylations; this result suggests that most of the DEGs do not recruit multiple types of histone demethylases together, but that, they instead recruit only a single type of histone demethylase. Our findings are consistent with previous biochemical studies in which the H3K9me3 demethylase formed a complex with the $\mathrm{H} 3 \mathrm{~K} 4$ methyltransferase and in reverse the H3K4me3 demethylases made a complex with the H3K9 methyltransferase.

Consequently, for genes that recruited complexes of the H3K9me3 demethylase-H3K4 methyltransferase, hypoxia can result in the predominant association of the DEGs with an increase of H3K9me3 without changes of the H3K4me3. Furthermore, by using the MotifLocator analyses, we identified 75 TFs whose binding motifs were significantly enriched in the
DMRs of the genes. Among the $75 \mathrm{TFs}$, we performed the ChIP assays for the positive target genes of the six TFs (JUN, ARNT, CREB1, HIF1A, E2F4, and RELA), that have previously reported associations with the JMJDs. Interestingly, however, several of them showed different binding strengths to the target genes between normoxia and hypoxia: hypoxia increased bindings of ARNT and HIF1A to the target genes with increase of H3K4me3. On the other hand, hypoxia did not change binding of CREB of the target genes with increase H3K9me3.

These results suggest that the TFs showing similar binding strengths to their target genes under hypoxia might be under the 'metabolic control'; by contrast, the TFs showing different binding strengths might be under the 'signaling control'. Based on this notion, the types of the TFs, together with the association between the gene expression and the prominent single-type histone methylation, can further characterize whether the single-type histone methylation can be determined by the environmental signaling. Or conversely, it could also clarify whether it could be affected by the metabolic states associated with the amounts of metabolites $\left(\mathrm{O}_{2}, \alpha-K G\right.$, and/or vitamin $\mathrm{C}$ ), which can also affect the activities of the histone demethylases.

\section{ACKNOWLEDGEMENTS}

This work was supported by the 2016 Research Fund of the University of Seoul for Hyunsung Park. 